\title{
INTEGRATION WITH RESPECT TO A VECTOR MEASURE AND FUNCTION APPROXIMATION
}

\author{
L. M. GARCÍA-RAFFI, D. GINESTAR, AND E. A. SÁNCHEZ-PÉREZ
}

Received 13 May 2000

The integration with respect to a vector measure may be applied in order to approximate a function in a Hilbert space by means of a finite orthogonal sequence $\left\{f_{i}\right\}$ attending to two different error criterions. In particular, if $\Omega \in \mathbb{R}$ is a Lebesgue measurable set, $f \in L_{2}(\Omega)$, and $\left\{A_{i}\right\}$ is a finite family of disjoint subsets of $\Omega$, we can obtain a measure $\mu_{0}$ and an approximation $f_{0}$ satisfying the following conditions: (1) $f_{0}$ is the projection of the function $f$ in the subspace generated by $\left\{f_{i}\right\}$ in the Hilbert space $f \in L_{2}\left(\Omega, \mu_{0}\right)$. (2) The integral distance between $f$ and $f_{0}$ on the sets $\left\{A_{i}\right\}$ is small.

\section{Introduction}

Let $\Omega \subset \mathbb{R}$ be a bounded Lebesgue measurable set. We consider the Hilbert space of functions $L_{2}(\Omega)$. A finite set of orthonormal functions $B=\left\{f_{i}\right\}_{i=1}^{n}$ in this space, generates a finite-dimensional subspace of $L_{2}(\Omega)$, F F . Given a function $f \in L_{2}(\Omega)$, we can obtain an approximation of $f$ as the projection of $f$ onto $\mathscr{F}$, that is, $f$ can be approximated as

$$
f \approx P(f)=\sum_{i=1}^{n} \alpha_{i} f_{i},
$$

where the coefficients $\alpha_{i}$ are given by

$$
\alpha_{i}=\left\langle f_{i}, f\right\rangle, \quad i=1, \ldots, n .
$$

Depending on the particular measure that defines the Hilbert space and the set of functions $B$ chosen, the approximation of $f,(1.1)$, is better or worse with respect to a given error criterion. However, the measure of the Hilbert space (and therefore the particular metric) is fixed when we define the framework we are going to work with. For example, suppose that we have an orthonormal system $\left\{f_{i}: i=1, \ldots, n\right\}$ in $L_{2}([0,1])$ and we want to approximate a function of $L_{2}([0,1])$ as the projection in the subspace $\operatorname{span}\left\{f_{i}: i=1, \ldots, n\right\}$. Then we can calculate easily the set of coefficients of this 
projection, but we cannot relate this set with the set of coefficients that we get if we change, for instance, the Lebesgue measure $\mu$ by another weighted measure $g \mu$. Since the error criterion usually depends on the particular metric of the Hilbert space, the solution of the problem strongly depends on the measure that we use in the definition of the Hilbert space.

This problem motivates the introduction of the integration with respect to a vector measure as a tool for function approximations. Up to a point, this framework allows us to introduce the measure that defines the function space as a variable of the problem, and then to attend to two or more error criterions in the approximation of a function in a finite-dimensional subspace. Although the theory of the spaces of integrable functions with respect to a vector measure has been strongly developed in the last thirty years, [2, 3, 7, 9], as far as we know, it has not been applied in the context of function approximations. In this paper, we present an application. In particular, we show how this framework could be useful to solve the following problem: suppose that we have a finite orthonormal sequence and we want to approximate a function $f$ as a linear combination of the elements of this sequence using the structure of a Hilbert space. Moreover, we want the integral of our approximation $f_{0}$ to be close to the integral of the function $f$ in certain subsets $\left\{A_{i}\right\}_{i=1}^{n}$, that is, we want the integral distance defined in the sets $\left\{A_{i}\right\}_{i=1}^{n}$ between $f$ and $f_{0}$ to be small. In this case, we can treat this problem in the following way. First, for each measure $\mu_{i}$ of a certain family we may obtain the projection of $f$ on the finite-dimensional space $\operatorname{span}\left\{f_{i}: i=1, \ldots, n\right\}$ of $L_{2}\left(\Omega, \mu_{i}\right)$, the Hilbert space endowed with the metric defined by $\mu_{i}$. Then, we can use $\mu_{i}$ as the variable of the problem, in order to find the best measure $\mu_{i}$ that also minimizes the integral distance.

In this paper, we show a procedure to obtain such an approximation in the mentioned theoretical context. Of course, all the elements that we have used in the above argument (the different errors we work with, the family of measures $\mu_{i}$, and the integral distance) must be defined in a precise and natural way. This will be done in Sections 2 and 3. Section 4 is devoted to the proof of the main theorem of the geometric procedure that we propose, and Section 5 provides several examples of applications of our formalism. Finally, we give in Section 6 some conclusions.

As the reader will see, the framework of the integration with respect to a vector measure is not necessary to obtain some of our results, since we only develop the theory in the finite-dimensional case (the image of the vector measure is defined in a finite-dimensional space) and for a special kind of vector measures. Anyway, there are two reasons to use it.

(1) The theory that we get in this way lets us define several metrics in the function space in a natural way, and leads to an easy formalism.

(2) It would be possible to extend our results to every vector measure with image in a Hilbert space, following the results that are known for spaces of integrable functions with respect to vector measures (see $[2,3,7]$ ).

However, although the results in this paper may be generalized in several directions, we prefer to sacrifice the generality to give our definitions and procedures in a concrete form in order to show that it is possible to obtain some tools to perform calculations. 
Therefore, we will only use the following particular kind of vector measures. Let $(\Omega, \Sigma)$ be the $\sigma$-algebra of the Lebesgue measurable sets of $\mathbb{R}$ and let $\mu$ be the Lebesgue measure. Let $\left(A_{i}\right)_{i=1}^{n}$ be a family of pairwise disjoint Lebesgue measurable subsets of $\mathbb{R}$ with (non null) finite measure. We define the vector measure

$$
\lambda: \Sigma \longrightarrow \mathbb{R}^{n}
$$

as $\lambda(A)=\sum_{i=1}^{n} \mu\left(A_{i} \cap A\right) e_{i}$, where $A \in \Sigma$ and $e_{i}, i=1, \ldots, n$ are the vectors of the canonical basis of $\mathbb{R}^{n}$.

Let $\Omega=\bigcup A_{i}$. Following the theory of integration with respect to a vector measure (see [7] and [4, Chapter 1]), we define the integral of a function $f \in L_{2}(\Omega)$ with respect to $\lambda$ as the operator

$$
\int_{\Omega} f d \lambda=\sum_{i=1}^{n}\left(\int_{A_{i}} f d \mu\right) e_{i} .
$$

If $\|\cdot\|$ is a norm in $\mathbb{R}^{n}$, we may obtain a norm related to this integral in order to construct a Banach space of (real) integrable functions. For instance, if we consider the $l_{1}$ norm in $\mathbb{R}^{n}$, then the expression $\left\|\int_{\Omega} f^{2} d \lambda\right\|_{1}^{1 / 2}$ gives the norm in $L_{2}(\Omega)$. Moreover, if $v=\left(v_{1}, \ldots, v_{n}\right)$ is a vector and $\langle\cdot, \cdot\rangle$ is the canonical inner product in $\mathbb{R}^{n}$, the expression

$$
\left\langle v, \int_{\Omega} f^{2} d \lambda\right\rangle^{1 / 2}
$$

is a norm if and only if $\left\langle v, e_{i}\right\rangle>0$ for each $i=1, \ldots, n$ which is equivalent to the natural norm of $L_{2}(\Omega)$ (see [1]). Throughout this paper, we consider the class of all these norms for different vectors $v \in \mathbb{R}^{n}$ satisfying $\left\langle v, e_{i}\right\rangle>0, i=1, \ldots, n$.

We use standard linear algebra and function spaces notation. The reader can find all the results that are needed about Banach spaces, lattices, and function spaces in $[6,8]$ or [10]. In particular, if $n$ is a natural number, then we write $\|\cdot\|_{l_{2}^{n}(v)}$ to denote the 2-norm with measure $v$ in the space $\mathbb{R}^{n}$, that is,

$$
\left\|\left(v_{i}\right)\right\|_{l_{2}^{n}(v)}=\left(\sum_{i=1}^{n} v_{i} v_{i}^{2}\right)^{1 / 2},
$$

where $v=\left(v_{1}, \ldots, v_{n}\right)$.

\section{Vector measure orthogonality: definitions and basic results}

Definition 2.1. Let $f, g \in L_{2}(\Omega)$. We say that they are orthogonal with respect to the vector measure $\lambda$ ( $\lambda$-orthogonal for short) if

$$
\int_{\Omega} f g d \lambda=0
$$

This condition is obviously equivalent to the fact that for each vector $v \in \mathbb{R}^{n}$, $\left\langle v, \int_{\Omega} f g d \lambda\right\rangle=0$. It means that the functions $f$ and $g$ are orthogonal when restricted to each $A_{i}$ in the definition of the vector measure $\lambda$. 
In the following, we will define our functional structure, which is related to special $\lambda$-orthogonal basis, and we will study some of its properties.

Definition 2.2. Let $B=\left\{f_{i}\right\}_{i=1}^{n} \subset L_{2}(\Omega)$ a set of non-(almost everywhere null) $\lambda$ orthogonal functions. We say that it is a $\lambda$-basis if the set $B_{\lambda}=\left\{\int_{\Omega} f_{i}^{2} d \lambda\right\}_{i=1}^{n}$ is a basis of the linear space $\mathbb{R}^{n}$.

For each basis $V$ of a space $\mathbb{R}^{n}$, we can define an inner product $\langle\cdot, \cdot\rangle_{V}$ such that $V$ is an orthonormal basis. If $C$ is the canonical basis of $\mathbb{R}^{n}$, the Gram matrix that represents this product when we work with the canonical coordinates of the vectors is

$$
(C)_{V}^{T}(C)_{V},
$$

where $(C)_{V}$ is the matrix of change of coordinates from the basis $C$ to the basis $V$.

Definition 2.3. We say that a couple

$$
\Lambda=\left(B, \lambda: \Sigma \longrightarrow \mathbb{R}^{n}\right)
$$

is a $\lambda$-approximation structure, where $B$ is a $\lambda$-basis of functions, and we have considered in $\mathbb{R}^{n}$ the scalar product $\langle\cdot, \cdot\rangle_{B_{\lambda}}$.

Proposition 2.4. Let $\Lambda=(B, \lambda)$ be a $\lambda$-approximation structure. Then, if $\left(v_{1}, \ldots, v_{n}\right)$ $\in \mathbb{R}^{n}$ are the coordinates of a vector $v$ in the basis $B_{\lambda}$ and $f=\sum_{i=1}^{n} \alpha_{i} f_{i}$, then

$$
\left\langle v, \int_{\Omega} f^{2} d \lambda\right\rangle_{B_{\lambda}}=\sum_{i=1}^{n} v_{i} \alpha_{i}^{2}
$$

Therefore, if $g=\sum_{i=1}^{n} \beta_{i} f_{i}$ and $\beta_{i} \neq 0$ for each $i=1, \ldots, n$, then

$$
\left\langle\int_{\Omega} g^{2} d \lambda, \int_{\Omega} f^{2} d \lambda\right\rangle_{B_{\lambda}}=\sum_{i=1}^{n} \alpha_{i}^{2} \beta_{i}^{2}=\left\|\left(\alpha_{i}\right)\right\|_{l_{2}^{n}\left(\beta^{2}\right)}^{2},
$$

where $\beta^{2}=\left(\beta_{1}^{2}, \ldots, \beta_{n}^{2}\right)$. In particular, if $f_{0}=\sum_{i=1}^{n} f_{i}$, then

$$
\left\langle\int_{\Omega} f_{0}^{2} d \lambda, \int_{\Omega} f^{2} d \lambda\right\rangle_{B_{\lambda}}=\sum_{i=1}^{n} \alpha_{i}^{2}=\|f\|_{L_{2}(\Omega)}^{2}
$$

for each $f \in \operatorname{span}\{B\}$.

Proposition 2.5. Let $f, g, h, k \in \operatorname{span}\{B\}$. Then

$$
\left\langle\int_{\Omega} f g d \lambda, \int_{\Omega} h k d \lambda\right\rangle_{B_{\lambda}}=\left\langle\int_{\Omega} f h d \lambda, \int_{\Omega} g k d \lambda\right\rangle_{B_{\lambda}} .
$$

Therefore, if $g=\sum_{i=1}^{n} \beta_{i} f_{i}$ and $f=\sum_{i=1}^{n} \alpha_{i} f_{i}$ are elements of $\operatorname{span}\{B\}$ and $\beta_{i} \neq 0$ for each $i=1, \ldots, n$, then

$$
\left\|\int_{\Omega} f g d \lambda\right\|=\left\|\left(\alpha_{i}\right)\right\|_{l_{2}^{n}\left(\beta^{2}\right)} .
$$


We want to remark that once we have fixed $v \in \mathbb{R}^{n}$ such that $\left\langle v, e_{i}\right\rangle>0, i=1, \ldots, n$, we can obtain a projection of a function $g \in L_{2}(\Omega)$ onto the subspace $\operatorname{span}\{B\}$ as

$$
P_{v}(g)=\sum_{i} \alpha_{i}(v) f_{i}
$$

where

$$
\alpha_{i}(v)=\frac{\left\langle v, \int_{\Omega} f_{i} g d \lambda\right\rangle_{B_{\lambda}}}{\left\langle v, \int_{\Omega} f_{i}^{2} d \lambda\right\rangle_{B_{\lambda}}} .
$$

This projection, $P_{v}(g)$, gives an approximation of $g$ over $\Omega$ in the Hilbert space sense.

Definition 2.6. We denote by $f_{0}$ the function $\sum_{i=1}^{n} f_{i}$. Let $g \in L_{2}(\Omega)$. The function $\Lambda_{\mathrm{a}}(g) \in \operatorname{span}\left\{f_{i}: i=1, \ldots, n\right\}$ that satisfies the equation

$$
\int_{\Omega} g d \lambda=\int_{\Omega} \Lambda_{\mathrm{a}}(g) f_{0} d \lambda,
$$

is called the approximation in area to the function $g$ in the $\lambda$-approximation structure.

It is obvious that the family of constants $\beta_{i}, i=1, \ldots, n$, defining $\Lambda_{\mathrm{a}}(g)=\sum_{i=1}^{n}$ $\beta_{i} f_{i}$ is unique, since $\left\{\int_{\Omega} f_{i}^{2} d \lambda\right\}_{i=1}^{n}$ is a basis. Moreover,

$$
\beta_{i}=\left\langle\int_{\Omega} g d \lambda, \int_{\Omega} f_{i}^{2} d \lambda\right\rangle_{B_{\lambda}} .
$$

We use the notation $\beta_{i}(g)$ for these coefficients if it were necessary to specify the function $g$.

If $\Lambda$ is a $\lambda$-approximation structure and $g$ is an element of $L_{2}(\Omega)$, we define the elements of the matrix $B_{g}=\left(b_{i j}\right)$ as

$$
b_{i j}=\left\langle\int_{\Omega} f_{i} g d \lambda, \int_{\Omega} f_{j}^{2} d \lambda\right\rangle_{B_{\lambda}} .
$$

Then, if we consider a function $f=\sum_{i=1}^{n} \gamma_{i} f_{i}$, we have

$$
\begin{aligned}
\int_{\Omega} \Lambda_{\mathrm{a}}(f g) f_{0} d \lambda & =\int_{\Omega}\left(\sum_{i=1}^{n} \gamma_{i} f_{i}\right) g d \lambda=\sum_{i=1}^{n} \gamma_{i}\left(\sum_{j=1}^{n} b_{i j} \int_{\Omega} f_{j}^{2} d \lambda\right) \\
& =\int_{\Omega}\left(\gamma_{1}, \ldots, \gamma_{n}\right) B_{g}\left(f_{1}^{2}, \ldots, f_{n}^{2}\right)^{T} d \lambda,
\end{aligned}
$$

where we have used matrix notation also for the vector of the functions $f_{j}^{2}$. Hence, the approximation in area of the function $f g$ is given by

$$
\Lambda_{\mathrm{a}}(f g)=\left(\gamma_{1}, \ldots, \gamma_{n}\right) B_{g}\left(f_{1}, \ldots, f_{n}\right)^{T} .
$$

Now, we consider a vector $v=\sum_{j=1}^{n} \omega_{j} \int_{\Omega} f_{j}^{2} d \lambda$ such that $\left\langle v, e_{i}\right\rangle_{B_{\lambda}}>0$ for all the vectors $e_{i}, i=1, \ldots, n$, of the canonical basis of $\mathbb{R}^{n}$. Such a vector $v$ exists, since $B$ is 
a basis, and defines a (positive) measure in $\Omega$. Then, the coefficients of the projection $P_{v}(g)$ of the function $g$ in the $\operatorname{subspace} \operatorname{span}\{B\}$ are

$$
\alpha_{j}(v)=\frac{\left\langle v, \int_{\Omega} f_{j} g d \lambda\right\rangle_{B_{\lambda}}}{\left\langle v, \int_{\Omega} f_{j}^{2} d \lambda\right\rangle_{B_{\lambda}}}=\alpha_{j}(v)=\frac{\sum_{i=1}^{n} \omega_{i}\left\langle\int_{\Omega} f_{i}^{2} d \lambda, \int_{\Omega} f_{j} g d \lambda\right\rangle_{B_{\lambda}}}{\omega_{j}} .
$$

Thus, the projection of $g$ can be written as

$$
P_{v}(g)=\left(\omega_{1}, \ldots, \omega_{n}\right) B_{g}^{T}\left(\frac{f_{1}}{\omega_{1}}, \ldots, \frac{f_{n}}{\omega_{n}}\right)^{T} .
$$

Therefore, the same matrix $B_{g}$ can be used in order to obtain both the approximation $\Lambda_{\mathrm{a}}(f g)$ for each function $f \in \operatorname{span}\{B\}$ and all the projections of $g$ in the sense of the Hilbert spaces with different measures induced by the vectors $v, P_{v}(g)$.

Now, we try to obtain an approximation of a given function $g$ in the sense of the Hilbert space, $P_{v}(g)$, which is a good approximation of $g$ in an "area sense." To find the vector $v$ determining this approximation, we impose new conditions to the $\lambda$-approximation structures.

\section{Error bounds for function approximations}

From now on, to construct the $\lambda$-approximation structures we consider special sets of functions $B=\left\{f_{i}\right\}$ that satisfy the following conditions:

(1) $\left\{\int_{\Omega} f_{i}^{2} d \lambda: i=1, \ldots, n\right\}$ is a basis of $\mathbb{R}^{n}$, as in Definition 2.3.

(2) For each $f_{i} \in B, \int_{A_{j}} f_{i} d \mu=\int_{A_{j}} f_{i}^{2} d \mu, j=1, \ldots, n$.

Condition (2) is then obviously equivalent to the fact that for each function $f \in$ $\operatorname{span}\left\{f_{i}\right\}, \int_{\Omega} f_{0} f d \lambda=\int_{\Omega} f d \lambda$. Therefore, $f_{0}$ works as an identity in the subspace generated by $B$ if we consider the integration with respect to $\lambda$. This property will be very important to select a good definition of an error between the approximations $P_{v}(g)$ and $\Lambda_{\mathrm{a}}(g)$. In fact, we impose property (2) on the $\lambda$-approximation structures in order to get a reasonable and unified definition for this error. The optimization of this error will be, of course, the answer of the problem that we proposed at the end of the last section. Note that, we have at least two reasonable definitions for an error criterion.

On one hand, we have the error from the Hilbert space point of view,

$$
\epsilon(g)_{w}=\left\langle w, \int_{\Omega}\left(P_{v}(g)-\Lambda_{\mathrm{a}}(g)\right)^{2} d \lambda\right\rangle_{B_{\lambda}}^{1 / 2}
$$

for a vector $w$ satisfying the conditions $\left\langle w, e_{i}\right\rangle>0, i=1, \ldots, n$.

On the other hand, we can give the error in an area sense, that may be defined as

$$
\epsilon_{A}(g)_{v}:=\left\|\int_{\Omega}\left(P_{v}(g)-\Lambda_{\mathrm{a}}(g)\right) d \lambda\right\|_{B_{\lambda}} .
$$

The following proposition shows that these two errors are really the same one, when we consider the vector $w$ induced by the function $f_{0}$. 
Proposition 3.1. Let $g \in L_{2}(\Omega)$. For a given vector $v$, the approximation in the Hilbert space sense

$$
P_{v}(g)=\sum_{i=1}^{n} \alpha_{i}(v) f_{i}
$$

satisfies

$$
\begin{aligned}
\left\|\int_{\Omega} P_{v}(g) d \lambda-\int_{\Omega} g d \lambda\right\|_{B_{\lambda}} & =\left\langle\int_{\Omega} f_{0} d \lambda, \int_{\Omega}\left(P_{v}(g)-\Lambda_{\mathrm{a}}(g)\right)^{2} d \lambda\right\rangle_{B_{\lambda}}^{1 / 2} \\
& =\left(\sum_{i=1}^{n}\left(\alpha_{i}(v)-\beta_{i}(g)\right)^{2}\right)^{1 / 2}
\end{aligned}
$$

where $\sum_{i=1}^{n} \beta_{i} f_{i}=\Lambda_{\mathrm{a}}(g)$.

Proof. Since both functions $\Lambda_{\mathrm{a}}(g)$ and $P_{v}(g)$ are in $\operatorname{span}\{B\}$, we can write the difference between them as follows:

$$
P_{v}(g)-\Lambda_{\mathrm{a}}(g)=\sum_{i=1}^{n}\left(\alpha_{i}(v)-\beta_{i}(g)\right) f_{i} .
$$

The definition of the $\lambda$-approximation structures gives

$$
\int_{\Omega} f_{0}\left(P_{v}(g)-\Lambda_{\mathrm{a}}(g)\right) d \lambda=\int_{\Omega}\left(P_{v}(g)-\Lambda_{\mathrm{a}}(g)\right) d \lambda .
$$

Straightforward calculations using Propositions 2.4 and 2.5 give the result.

In the following, we will show a procedure to estimate the error defined in Proposition 3.1. The main idea is to find a bound for expression (3.4) with two different parts which can be associated with different geometric aspects of our function space.

We have that

$$
\begin{aligned}
\left\|\int_{\Omega} \sum_{i=1}^{n} \alpha_{i}(v) f_{i} d \lambda-\int_{\Omega} g d \lambda\right\|_{B_{\lambda}} \leq & \left\|\int_{\Omega} \sum_{i=1}^{n}\left(\alpha_{i}(v) f_{i}-g f_{0}\right) d \lambda\right\|_{B_{\lambda}} \\
& +\left\|\int_{\Omega} g\left(I-f_{0}\right) d \lambda\right\|_{B_{\lambda}},
\end{aligned}
$$

where we have denoted by $I$ the identity function $\chi_{\Omega}$. We intend to find a vector $v$ that minimizes the error

$$
\left(\sum_{i=1}^{n}\left(\alpha_{i}(v)-\beta_{i}(g)\right)^{2}\right)^{1 / 2},
$$

and this will be done by analyzing the two terms on the right-hand side of (3.7). 
Definition 3.2. We define the symmetry error associated to a function $g$ and a vector $v$ in a $\lambda$-approximation structure as

$$
\epsilon_{S}(g, v)=\left\|\int_{\Omega}\left(\sum_{i=1}^{n} \alpha_{i}(v) f_{i}-g f_{0}\right) d \lambda\right\|_{B_{\lambda}},
$$

and the orthogonality error associated to the function $g$ as

$$
\epsilon_{o}(g):=\left\|\int_{\Omega} g\left(I-f_{0}\right) d \lambda\right\|_{B_{\lambda}} .
$$

Note that the symmetry error depends on the vector $v$, but this is not the case for the orthogonality error, that only depends on the orthogonality properties of the function $g$ with respect to the $\lambda$-approximation structure. We write simply $\epsilon_{S}(g)$ for the symmetry error if the dependence of the vector $v$ is clear.

3.1. The symmetry error. As we have shown in Section 2, the coefficients of the projection of a function $g$ in the Hilbert space defined by the vector $v$ are given by

$$
\alpha_{i}(v)=\frac{\left\langle v, \int_{\Omega} f_{i} g d \lambda\right\rangle_{B_{\lambda}}}{v_{i}}
$$

where the components of $v$ in the basis $B_{\lambda}$ are given by

$$
v_{i}=\left\langle v, \int_{\Omega} f_{i} d \lambda\right\rangle_{B_{\lambda}} .
$$

Although this is only defined in the classical situation for vectors $v$ that define a positive measure in the related Hilbert space (that is, $\left\langle v, e_{i}\right\rangle>0, i=1, \ldots, n$ ), we can extend this definition for the general case of any vector $v \in \mathbb{R}^{n}$ using the same formula. Of course, we need to give a definition of $\alpha_{i}(v)$ when $v_{i}=0$. We can write

$$
\Lambda_{\mathrm{a}}\left(f_{0} g\right)=\sum_{i} \beta_{i}\left(f_{0} g\right) f_{i} .
$$

The definition of the coefficients $\beta_{i}\left(f_{0} g\right)$ (see Definition 2.6) motivates that in the case that $v_{i}=0$, we define $\alpha_{i}(v)=\beta_{i}\left(f_{0} g\right)$.

We see that it is possible to find for each function $g$ a (nontrivial) vector $v=$ $\left(v_{1}, \ldots, v_{n}\right)$ such that the expression

$$
\sum_{i=1}^{n} v_{i}^{2}\left(\alpha_{i}(v)-\beta_{i}\left(f_{0} g\right)\right)^{2}
$$

is equal to 0 . This result means that it is possible to find a (non necessarily positive) measure induced by the vector $v$, such that $\alpha_{i}(v)=\beta_{i}\left(f_{0} g\right)$ for every $i=1, \ldots, n$.

For the sake of clarity, we introduce the following notation. 
Definition 3.3. If $f, g, h, k \in €_{2}(\Omega)$, we define the commutator $[f, g \mid h, k]$ as

$$
[f, g \mid h, k]:=\left\langle\int_{\Omega} f g d \lambda, \int_{\Omega} h k d \lambda\right\rangle_{B_{\lambda}}-\left\langle\int_{\Omega} h g d \lambda, \int_{\Omega} f k d \lambda\right\rangle_{B_{\lambda}} .
$$

Note that the commutator is linear for each component. In particular, this means that, as a consequence of the definition of the coefficients $\alpha_{i}(v)$ and $\beta_{i}\left(f_{0} g\right)$ related to a function $g$ and a vector $v$ of components $v_{i}$ in the basis $B_{\lambda}$, we have that

$$
\begin{aligned}
{\left[f_{0}, \sum_{j}^{n} v_{j} f_{j} \mid f_{i}, g\right]=} & \left\langle\int_{\Omega} f_{0}\left(\sum_{j} v_{j} f_{j}\right) d \lambda, \int_{\Omega} f_{i} g d \lambda\right\rangle_{B_{\lambda}} \\
& -\left\langle\int_{\Omega} f_{i}\left(\sum_{j} v_{j} f_{j}\right) d \lambda, \int_{\Omega} f_{0} g d \lambda\right\rangle_{B_{\lambda}} \\
= & \sum_{j} v_{j}\left(\int_{\Omega} f_{j}^{2} d \lambda, \int_{\Omega} f_{i} g d \lambda\right\rangle_{B_{\lambda}}-v_{i}\left\langle\int_{\Omega} f_{i}^{2} d \lambda, \int_{\Omega} f_{0} g d \lambda\right\rangle_{B_{\lambda}} \\
= & v_{i}\left(\alpha_{i}(v)-\beta_{i}\left(f_{0} g\right)\right)
\end{aligned}
$$

for each $i=1, \ldots, n$. Considering the error vector $\left(v_{1}\left(\alpha_{1}(v)-\beta_{1}\right), \ldots, v_{n}\left(\alpha_{n}(v)-\beta_{n}\right)\right)$, if we define the commutator matrix as $A_{\text {com }}=\left(a_{i j}\right)$ as

$$
a_{i j}=\left[f_{0}, f_{i} \mid f_{j}, g\right]
$$

we have

$$
\left(v_{1}\left(\alpha_{1}(v)-\beta_{1}\right), \ldots, v_{n}\left(\alpha_{n}(v)-\beta_{n}\right)\right)=\left(v_{1}, \ldots, v_{n}\right) A_{\mathrm{com}} .
$$

But the matrix $A_{\text {com }}$ is always singular, since

$$
\sum_{j} a_{i j}=\sum_{j}\left[f_{0}, f_{i} \mid f_{j}, g\right]=\left[f_{0}, f_{i} \mid f_{0}, g\right]=0,
$$

for every $i=1, \ldots, n$.

This means that we can always find a nontrivial vector $v$ that makes the symmetry error equal to zero.

Note that, if we can assure that the vector $v$ has positive coordinates (in the canonical basis), we get a positive measure and then a solution from a Hilbert space point of view. This condition can be written using convex analysis arguments, since we just need to assure that the vector 0 is in the convex closure of the file vectors of the transformation of the matrix $A_{\text {com }}$ for the canonical basis. For example, we can use the geometric form of the Hahn-Banach theorem to get easy characterizations of this result (see $[1,5]$ ), and would be also applied in the general context of a vector measure defined on a Banach space.

We can easily obtain other consequences of the former argument. For example, if we get a symmetric matrix $A_{\text {com }}$ for a particular problem, we can assure that we have 
a complete solution, since the vector $v=(1 / n, \ldots, 1 / n)$ defining a positive measure is in the kernel of $A_{\text {com }}$. This is the reason which motivates the use of the expression symmetry error.

3.2. The orthogonality error. As we have said in the definition of the orthogonality error associated to the function $g$, this error does not depend on the vector $v$. This means that this error is only related to the adequation of the chosen basis to approximate the function $g$. As we can see, the error expression

$$
\epsilon_{o}(g)=\left\|\int_{\Omega} g\left(I-f_{0}\right) d \lambda\right\|_{B_{\lambda}}
$$

is small when the function $g$ is almost (vector) orthogonal to the function $\left(I-f_{0}\right)$. The assumed properties for the basis functions of the $\lambda$-approximation structure assures that the function $f_{0}$ is a projection of the identity function onto the subspace generated by the same basis. In fact, for each $i, j=1, \ldots, n$, we have

$$
\int_{A_{i}} f_{j}\left(I-f_{0}\right) d \mu=\int_{A_{i}}\left(f_{j}-f_{j}^{2}\right) d \mu=0,
$$

and then the function $\left(I-f_{0}\right)$ is (vector) orthogonal to each function of $\operatorname{span}\left\{f_{i}: i=\right.$ $1, \ldots, n\}$. Therefore, a $\lambda$-approximation structure is good to approximate a function $g$, when $g$ is almost in the subspace $\operatorname{span}\left\{f_{i}: i=1, \ldots, n\right\}$, and, in this case, the orthogonality error is small. It is also interesting (but only from the orthogonality error point of view) when the projection of $g$ in the complement of the subspace generated by the basis functions is (vector) orthogonal to $\left(I-f_{0}\right)$.

Anyway, we can obtain a general bound for the orthogonality error as a direct application of the theory of the Bochner integration [8]. Using the representation theorem for vector measures of the Radon-Nikodym theory, we know that of course the measure $\lambda$ is representable (see [4, Sections 1, 2, and 3]). This means that there is a Bochner integrable function $h$ such that for each measurable set $A \subset \Omega$,

$$
\lambda(A)=\int_{A} h d \mu
$$

In our case, the function is $h=\sum_{i=1}^{n} e_{i} \chi_{A_{i}}$, where $\chi_{A_{i}}$ is the characteristic function of the set $A_{i}$. This means that we can write for each function $g \in L_{2}(\Omega)$,

$$
\int_{\Omega} g d \lambda=\int_{\Omega} g h d \mu
$$

Now, we can apply the properties of the Bochner integral to the expression

$$
\epsilon_{o}(g)=\left\|\int_{\Omega} h g\left(I-f_{0}\right) d \mu\right\|_{B_{\lambda}} .
$$


An application of a well-known inequality for the Bochner integral (see [4, Theorem 4, Section II.2]) and the Hölder inequality lead us to

$$
\begin{aligned}
\epsilon_{o}(g) & \leq \int_{\Omega}\left\|h g\left(I-f_{0}\right)\right\|_{B_{\lambda}} d \mu \\
& \leq\left(\int_{\Omega}\left\|h\left(I-f_{0}\right)\right\|_{B_{\lambda}}^{2} d \mu\right)^{1 / 2}\left(\int_{\Omega}|g|^{2} d \mu\right)^{1 / 2} \\
& =C\|g\|_{L_{2}(\Omega)} .
\end{aligned}
$$

As we can see, we get a bound for $\epsilon_{o}(g)$ that depends on the norm $\|g\|_{L_{2}(\Omega)}$ and a constant $C$ that depends on the function $h\left(I-f_{0}\right)$. A direct calculation shows that

$$
C=\left(\int_{\Omega}\left\|h\left(I-f_{0}\right)\right\|_{B_{\lambda}}^{2} d \mu\right)^{1 / 2}=\left(\sum_{i=1}^{n}\left\|e_{i}\right\|_{B_{\lambda}}^{2} \int_{A_{i}}\left(I-f_{0}\right)^{2} d \mu\right)^{1 / 2}
$$

\section{Error-invariant perturbations on the original function $g$}

In this section, we find a subspace $S$ of $L_{2}(\Omega)$ that satisfies that, if $g$ is the original function and $g_{1} \in S$, then $\epsilon_{S}\left(g+g_{1}\right)=\epsilon_{S}(g)$ and $\epsilon_{o}\left(g+g_{1}\right)=\epsilon_{o}(g)$. We need one more definition. Suppose as in Section 3 that we work in the context of a particular $\lambda$-approximation structure. We use the same notation.

Definition 4.1. Let $V$ be a subspace of $L_{2}(\Omega)$. Then we define the following subset of $L_{2}(\Omega)$,

$$
V^{\lambda}=\left\{f \in L_{2}(\Omega): \int_{\Omega} f g d \lambda=0, \forall g \in V\right\}
$$

It is easy to see that $V^{\lambda}$ is a (closed) subspace of $L_{2}(\Omega)$.

The following result give the right range of application of our geometric arguments. As a corollary, we characterize which are the perturbations $g_{1}$ on the original function $g$ such that the measure that minimizes the symmetry and orthogonal errors for $g$ also minimizes these errors for $g+g_{1}$.

THeorem 4.2. Let $S=\operatorname{span}\left\{f_{1}, \ldots, f_{n}\right\}+\operatorname{span}\left\{I, f_{1}, \ldots, f_{n}\right\}^{\lambda}$. Then

(1) The sum of subspaces in the definition of $S$ is a direct sum.

(2) For each $g_{1} \in S, \epsilon_{o}\left(g_{1}\right)=0$, and $\epsilon_{S}\left(g_{1}\right)=0$.

Proof. First we show (1). If $f \in \operatorname{span}\left\{f_{1}, \ldots, f_{n}\right\} \cap \operatorname{span}\left\{I, f_{1}, \ldots, f_{n}\right\}^{\lambda}$, we can write it as $f=\sum_{i=1}^{n} \alpha_{i} f_{i}$. Moreover, for each $i=1, \ldots, n$ we have

$$
\int_{\Omega_{0}} f f_{i} d \lambda=\alpha_{i} \int_{\Omega_{0}} f_{i}^{2} d \lambda=0
$$

Thus, since $\int_{\Omega_{0}} f_{i}^{2} d \lambda$ is not the null vector, we get $f=0$. 
To prove (2), let $g_{1} \in S$. Then $g_{1}=g_{a}+g_{b}$, where $g_{a} \in \operatorname{span}\left\{f_{1}, \ldots, f_{n}\right\}$ and $g_{b} \in$ $\operatorname{span}\left\{I, f_{1}, \ldots, f_{n}\right\}^{\lambda}$. We just need to show that $\epsilon_{s}\left(g_{a}\right)=\epsilon_{s}\left(g_{b}\right)=\epsilon_{o}\left(g_{a}\right)=\epsilon_{o}\left(g_{b}\right)=0$. We write $g_{a}=\sum_{i=1}^{n} \alpha_{i} f_{i}$. Obviously, for each vector $v, P_{v}\left(g_{a}\right)=g_{a}$, and

$$
\int_{\Omega_{0}}\left(P_{v}\left(g_{a}\right)-g_{a} f_{0}\right) d \lambda=\int_{\Omega_{0}}\left(\sum_{i=1}^{n} \alpha_{i} f_{i}-\sum_{i=1}^{n} \alpha_{i} f_{i}^{2}\right) d \lambda=0,
$$

and then $\epsilon_{s}\left(g_{a}\right)=0$. Moreover, $\epsilon_{o}\left(g_{a}\right)=0$, since

$$
\int_{\Omega_{0}} g_{a}\left(I-f_{0}\right) d \lambda=\sum_{i=1}^{n} \alpha_{i} \int_{\Omega_{0}}\left(f_{i}-f_{i}^{2}\right) d \lambda=0 .
$$

For $g_{b}$, we get for each vector $v$ that $P_{v}\left(g_{b}\right)=0$, since $\left\langle v, \int_{\Omega_{0}} g_{b} f_{i} d \lambda\right\rangle=0$ for all $i=1, \ldots, n$. Moreover, $\int_{\Omega_{0}} g_{b} f_{0} d \lambda=0$. Then

$$
\int_{\Omega_{0}}\left(P_{v}\left(g_{b}\right)-g_{b} f_{0}\right) d \lambda=0
$$

and $\epsilon_{s}\left(g_{b}\right)=0$. Since $\left(I-f_{0}\right) \in \operatorname{span}\left\{I, f_{1}, \ldots, f_{n}\right\}$, it is obvious that $\epsilon_{o}\left(g_{b}\right)=0$.

CoRollary 4.3. If $g \in L_{2}(\Omega)$ and $g_{1} \in S$, then $\epsilon_{s}\left(g+g_{1}\right)=\epsilon_{s}(g)$ and $\epsilon_{o}\left(g+g_{1}\right)=$ $\epsilon_{o}(g)$.

\section{Some examples}

In this section, we apply the decomposition of the error bound (3.7) to show some possibilities of our $\lambda$-approximation structures. We want to find the best vector $v$ that makes zero the symmetry error for a function $g, \epsilon_{S}(g)$, that gives also a small value of the orthogonality error, $\epsilon_{o}(g)$. In the following examples we will see the adequation of a $\lambda$-approximation structure to find a good approximation for three different functions. In Example 5.1, we see that the basis is good enough, but if we want to improve the result by selecting a special vector $v$, the right solution gives a nonpositive measure. In Example 5.2, we show that the best vector $v$ is the one that defines the canonical Lebesgue measure in the set $\Omega$. However, we see in Example 5.3 that, if we introduce a little perturbation in the original function, the symmetric result that we got for the function in Example 5.2 does not preserve the area under the function in the special sets of the definition in the vector measure.

We select $\Omega$ as the interval $[0,6]$. The $\lambda$-approximation structure is constructed with six functions $f_{1}, \ldots, f_{6}$ and six intervals $[i-1, i], i=1, \ldots, 6$.

The vector measure $\lambda$ is defined as

$$
\lambda(A)=\sum_{i=1}^{6} e_{i} \mu(A \cap[i-1, i]),
$$

where $\mu(A)=\int_{A} d x$, and $A \in \Sigma$. We construct each one of the six functions as a polynomial of degree 2 defined in one of the interval and a polynomial of degree 4 
defined in the consecutive interval. Following the definition of our $\lambda$-structures, all these functions $f_{i}$ must satisfy

$$
\int_{\Omega} f_{i}^{2} d \lambda=\int_{\Omega} f_{i} d \lambda,
$$

and of course they must be (vector) orthogonal,

$$
\int_{\Omega} f_{i} f_{j} d \lambda=0 \quad \forall i \neq j
$$

We have chosen the following functions $f_{1}, \ldots, f_{6}$ satisfying the above conditions.

$$
\begin{aligned}
& f_{1}(x)=5 x(1-x) \chi_{[0,1]}+3(2-x)(x-1)\left(14 x^{2}-42 x+31\right) \chi_{[1,2]}, \\
& f_{2}(x)=3 x(1-x)\left(14 x^{2}-14 x+3\right) \chi_{[0,1]}+5(2-x)(x-1) \chi_{[1,2]}, \\
& f_{3}(x)=5(3-x)(x-2) \chi_{[2,3]}+3(4-x)(x-3)\left(14 x^{2}-98 x+171\right) \chi_{[3,4]}, \\
& f_{4}(x)=3(3-x)(x-2)\left(14 x^{2}-70 x+87\right) \chi_{[2,3]}+5(4-x)(x-3) \chi_{[3,4]}, \\
& f_{5}(x)=5(5-x)(x-4) \chi_{[4,5]}+3(6-x)(x-5)\left(14 x^{2}-154 x+423\right) \chi_{[5,6]}, \\
& f_{6}(x)=3(5-x)(x-4)\left(14 x^{2}-126 x+283\right) \chi_{[4,5]}+5(6-x)(x-5) \chi_{[5,6]}
\end{aligned}
$$

Plots of functions $f_{1}$ and $f_{2}$ are given in Figures 5.1 and 5.2. The functions $f_{3}$ and $f_{5}$ are translations of $f_{1}$ to the intervals $[2,4]$ and $[4,6]$, respectively. The functions $f_{4}$ and $f_{6}$ are defined as translations of $f_{2}$ to the intervals $[2,4]$ and $[4,6]$.

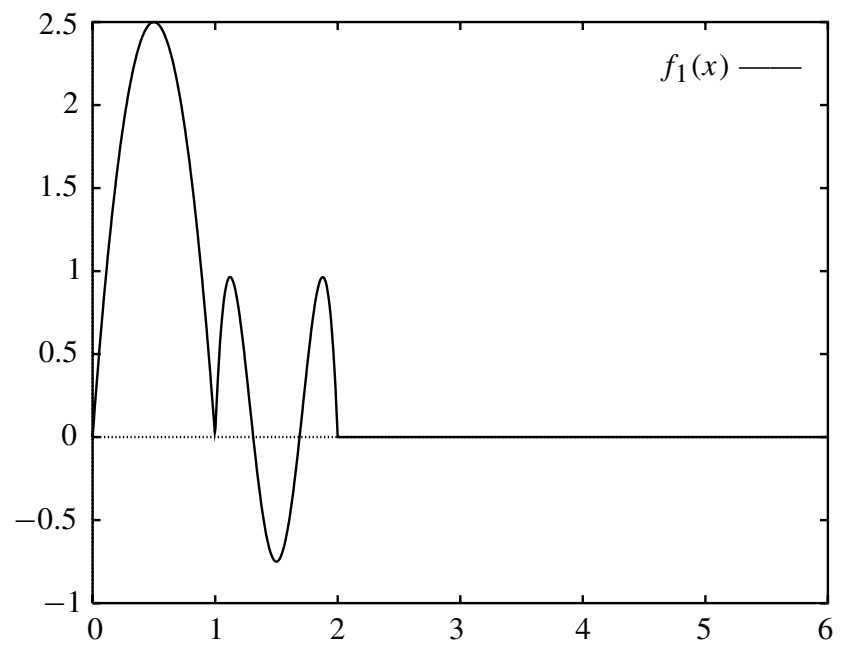

Figure 5.1. Plot of function $f_{1}$ of the $\lambda$-approximation structure. 


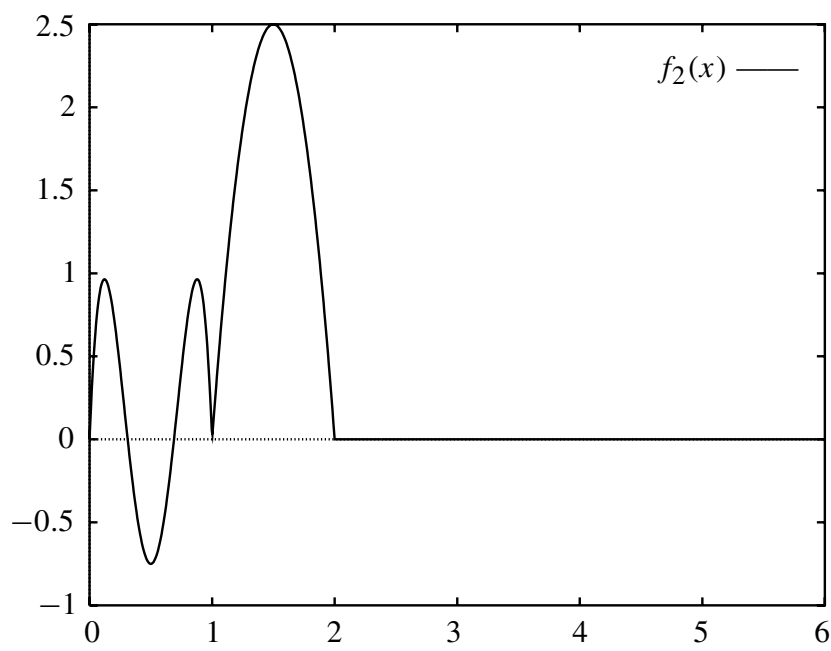

Figure 5.2. Plot of function $f_{2}$ of the $\lambda$-approximation structure.

Now, we need to calculate the matrix of the product $\langle\cdot, \cdot\rangle_{B_{\lambda}}$ with respect to the canonical basis. The (canonical) coordinates of the vectors $\int_{[0,6]} f_{i}^{2} d \lambda$ are

$$
\begin{gathered}
\int_{[0,6]} f_{1}^{2} d \lambda=\int_{[0,6]} f_{1} d \lambda=\left(\frac{5}{6}, \frac{1}{10}, 0,0,0,0\right), \quad \int_{[0,6]} f_{2} d \lambda=\left(\frac{1}{10}, \frac{5}{6}, 0,0,0,0\right), \\
\int_{[0,6]} f_{3} d \lambda=\left(0,0, \frac{5}{6}, \frac{1}{10}, 0,0\right), \quad \int_{[0,6]} f_{4} d \lambda=\left(0,0, \frac{1}{10}, \frac{5}{6}, 0,0\right), \\
\int_{[0,6]} f_{5} d \lambda=\left(0,0,0,0, \frac{5}{6}, \frac{1}{10}\right), \quad \int_{[0,6]} f_{6} d \lambda=\left(0,0,0,0, \frac{1}{10}, \frac{5}{6}\right) .
\end{gathered}
$$

Therefore, $(B)_{C}$ is the $6 \times 6$ block diagonal matrix

$$
B_{C}=\left(\begin{array}{ccc}
D_{1} & & \\
& D_{2} & \\
& & D_{3}
\end{array}\right)
$$

with three $2 \times 2$ blocks of the form

$$
D_{i}=\left(\begin{array}{cc}
\frac{5}{6} & \frac{1}{10} \\
\frac{1}{10} & \frac{5}{6}
\end{array}\right) .
$$

The Gram matrix of the scalar product is $\left(\left((B)_{C}^{-1}\right)^{T}\right)(B)_{C}^{-1}$, which has the same structure as $(B)_{C}$.

Example 5.1. In this example, we study the approximation to the function $g(x)=$ $\sin ^{3}(\pi x)$ in the interval $[0,6]$ with the former $\lambda$-approximation structure. As we can 
see in Figure 5.3, this is a good structure to approximate the function $g(x)$. The direct calculation of the orthogonality error gives

$$
\epsilon_{o}(g)=\left\|\int_{[0,6]} g\left(I-f_{0}\right) d \lambda\right\|_{B_{\lambda}}=0.0238 .
$$

This is a small value that can be accepted for this error. For the calculation of the symmetry error, we need the (canonical) coordinates of the vector integral of the functions $f_{i} g, i=0, \ldots, 6$ ( $f_{0}$ is defined as $\sum_{i=1}^{n} f_{i}$, as in the former sections).

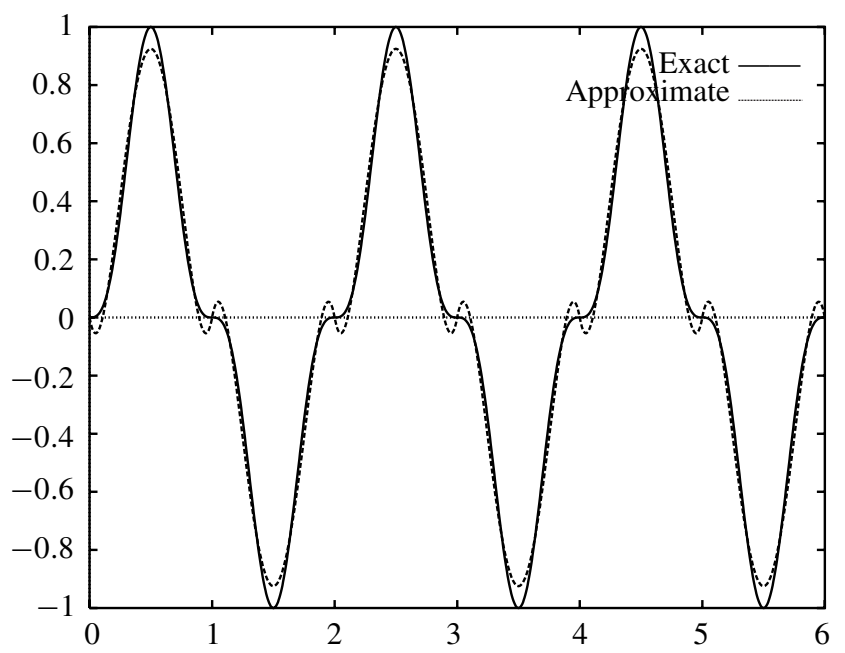

Figure 5.3. Plot of the function $g(x)$ and the approximation $P_{v}(g)(x)$.

These values are

$$
\begin{aligned}
& \int_{[0,6]} f_{0} g d \lambda=(0.4173,-0.4173,0.4173,-0.4173,0.4173,-0.4173), \\
& \int_{[0,6]} f_{1} g d \lambda=(0.4778,0.0605,0,0,0,0) \\
& \int_{[0,6]} f_{2} g d \lambda=(-0.0605,-0.4778,0,0,0,0) \\
& \int_{[0,6]} f_{3} g d \lambda=(0,0,0.4778,0.0605,0,0) \\
& \int_{[0,6]} f_{4} g d \lambda=(0,0,-0.0605,-0.4778,0,0) \\
& \int_{[0,6]} f_{5} g d \lambda=(0,0,0,0,0.4778,0.0605), \\
& \int_{[0,6]} f_{6} g d \lambda=(0,0,0,0,-0.0605,-0.4778) .
\end{aligned}
$$


Now, we compute the whole set of commutators that define the coefficients of the commutator matrix $A_{\text {com }}$. We get the $6 \times 6$ block diagonal matrix

$$
A_{\text {com }}=\left(\begin{array}{lll}
A_{1} & & \\
& A_{2} & \\
& & A_{3}
\end{array}\right)
$$

with the following $2 \times 2$ blocks

$$
A_{i}=\left(\begin{array}{ll}
0.0039 & -0.0039 \\
0.0039 & -0.0039
\end{array}\right)
$$

An element of the kernel of this commutator matrix is $(1,-1,1,-1,1,-1)$. This vector does not define a positive measure and it cannot be associated to an approximation from the Hilbert space point of view. Nevertheless, we can calculate the coefficients $\alpha_{i}(v)$ with this vector, and we get a good approximation $P_{v}(g)(x)$ to the function

$$
P_{v}(g)(x)=0.569\left(f_{1}(x)+f_{3}(x)+f_{5}(x)\right)-0.569\left(f_{2}(x)+f_{4}(x)+f_{6}(x)\right) .
$$

Of course, in this case the symmetry error is 0 . The fact that the matrix $A_{\text {com }}$ has small coefficients means that $\epsilon_{s}$ will be (nonzero but) small even in the case that we get another vector for the calculus of the coefficients (e.g., the canonical measure $(1,1,1,1,1,1))$.

The conclusion of this example is therefore that we can get a good result for this function $g$ with our $\lambda$-approximation structure easily. If we take the exact solution $v=(1,-1,1,-1,1,-1)$, a bound for the error is

$$
\left\|\int_{[0,6]} \sum_{i=1}^{6} \alpha_{i}(v) f_{i} d \lambda-\int_{[0,6]} g d \lambda\right\|_{B_{\lambda}} \leq 0.0238+0 .
$$

The values of the integrals $\int_{[i-1, i]} g(x) d \mu$ and $\int_{[i-1, i]} P_{v}(g)(x) d \mu$ will be almost the same for each $i=1, \ldots, 6$.

Example 5.2. In this example, we use the same $\lambda$-approximation structure to obtain an approximation for the function

$$
g(x)=\sqrt{|\sin (2 \pi x)|} \chi_{[0,2]}-\sqrt{|\sin (2 \pi x)|} \chi_{[2,4]}+\sqrt{|\sin (2 \pi x)|} \chi_{[4,6]} .
$$

After the calculation of all the integrals $\int_{[0,6]} f_{i} g d \lambda, i=0, \ldots, 6$, we get the $6 \times 6$ block diagonal matrix

$$
A_{\mathrm{com}}=\left(\begin{array}{ccc}
A_{1} & 0 & 0 \\
0 & A_{2} & 0 \\
0 & 0 & A_{3}
\end{array}\right)
$$

with the following $2 \times 2$ blocks

$$
A_{1}=\left(\begin{array}{cc}
-0.02885 & 0.02885 \\
0.02885 & -0.02885
\end{array}\right), \quad A_{2}=-A_{1}, \quad A_{3}=A_{1} .
$$


An exact solution to this problem is given by the vector $v=(1,1,1,1,1,1)$, that defines the canonical measure. The calculation of the coefficients $\alpha_{i}(v)$ gives the approximation

$$
P_{v}(g)(x)=0.8140\left(f_{1}(x)+f_{2}(x)-f_{3}(x)-f_{4}(x)+f_{5}(x)+f_{6}(x)\right) .
$$

The symmetry error is then 0 . The orthogonality error takes the value $\epsilon_{o}(g)=0.0077$. Therefore, the canonical solution gives in this case a good approximation in area, which means that the integrals of $g$ and $P_{v}(g)(x)$ are very similar in each subinterval $[i-1, i]$, $i=1, \ldots, 6$. As we can see in Figure 5.4, it does not mean that we have what we usually call "a good approximation." The functions $g$ and $P_{v}(g)(x)$ are in fact rather different.

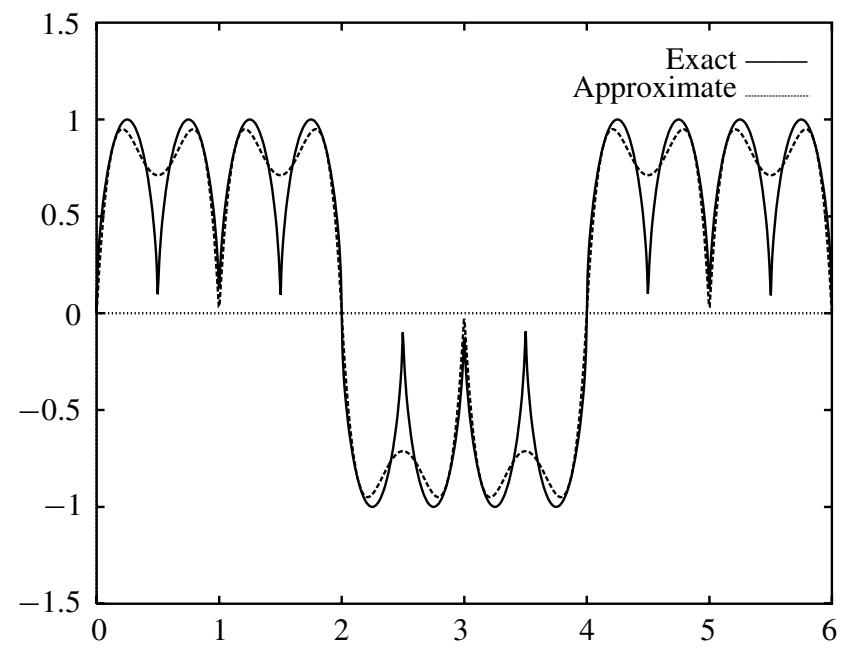

Figure 5.4. Plot of the function $g(x)$ and the approximation $P_{v}(g)(x)$ for Example 5.2.

Example 5.3. We are going to introduce a perturbation in the function $g(x)$ of the former example. Suppose that $g(x)$ is a signal that is affected by a new term of the form

$$
r(x)=\frac{5}{3}(x-1) x \chi_{[0,1]} .
$$

Thus, we want to find the best measure for the function $k(x)=g(x)+r(x)$. The orthogonality error is the same in this case as in the former one, $\epsilon_{o}(k)=0.0077$, since $r(x)$ is $\lambda$-orthogonal to the function $\left(I-f_{0}\right)$. However, the symmetry error is not the same. The matrix $A_{\text {com }}$ is again a block diagonal matrix with the same structure as (5.15) with the blocks

$$
A_{1}=\left(\begin{array}{cc}
-0.02885 & 0.02885 \\
0.06943 & -0.06943
\end{array}\right), \quad A_{2}=\left(\begin{array}{cc}
0.02885 & -0.02885 \\
-0.02885 & 0.02885
\end{array}\right), \quad A_{3}=-A_{2} \text {. }
$$


A vector of the kernel of $A_{\text {com }}$ is $(1,0.41558,1,1,1,1)$. The vector that we got in the former example is not good in this case. If we obtain the coordinates of this vector in the canonical basis and we normalize it to get a measure in the interval $[0,6]$, we obtain the solution

$$
v_{1}=(1.0385,0.5298,1.1079,1.1079,1.1079,1.1079) .
$$

Using this measure to calculate the coefficients $\alpha_{i}\left(v_{1}\right)$, we obtain the approximation

$$
\begin{aligned}
P_{v_{1}}(k)(x)= & 0.4759 f_{1}(x)+0.8547 f_{2}(x)-0.8141\left(f_{3}(x)+f_{4}(x)\right) \\
& +0.8141\left(f_{5}(x)+f_{6}(x)\right) .
\end{aligned}
$$

The solution that we get for the canonical measure $v_{2}=(1,1,1,1,1,1)$ is

$$
P_{v_{2}}(k)(x)=0.5165 f_{1}(x)+0.8141\left(f_{2}(x)-f_{3}(x)-f_{4}(x)+f_{5}(x)+f_{6}(x)\right) .
$$

In Figure 5.5, we show the plots of the functions $k(x), P_{v_{1}}(k)(x)$, and $P_{v_{2}}(k)(x)$ in the subinterval $[0,2]$. We can see that the approximation is not very good in any case, and the difference between $P_{v_{1}}(k)$ and $P_{v_{2}}(k)$ does not seem to be very important. Anyway, $P_{v_{1}}(k)$ is a good approximation in area (the integral distance is small in each subinterval $[i, i-1], i=1, \ldots, 6)$ to the function $k$ and $P_{v_{2}}(k)$ is not. To show this, we compute in the following the values of the integral of the differences between the function $k$ and the approximations $P_{v_{1}}(k)$ and $P_{v_{2}}(k)$ in the subintervals $[0,1]$ and $[1,2]$. We also calculate the relative error in each case (the integral of the difference divided by the value of the integral of $k(x)$ in each subinterval).

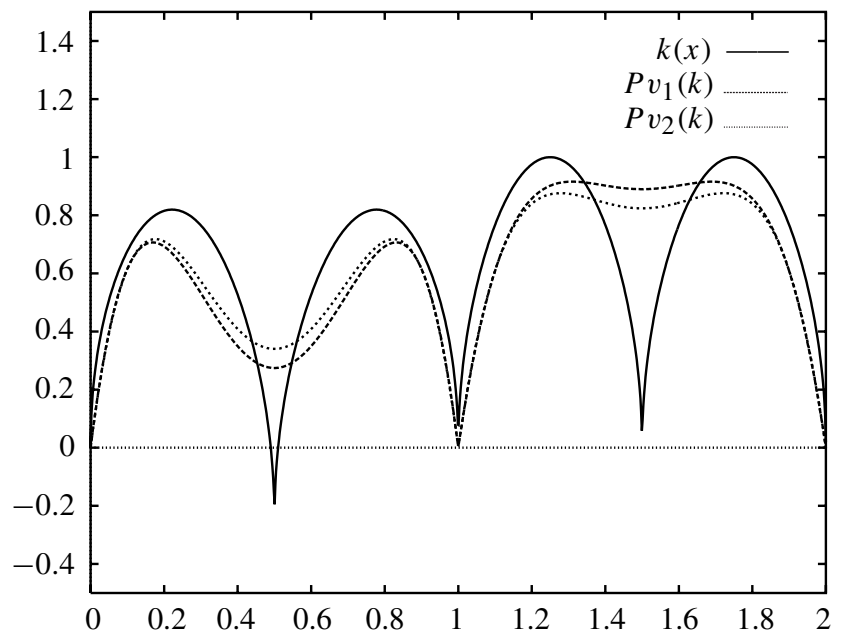

Figure 5.5. Plot of the functions $k(x), P_{v_{1}}(k)(x)$, and $P_{v_{2}}(k)(x)$ for Example 5.3. 
We obtain the following results:

$$
\begin{array}{ll}
\int_{[0,1]}\left(P_{v_{1}}(k)(x)-k(x)\right) d \mu=-0.0029 & \text { Error }=0.6 \%, \\
\int_{[0,1]}\left(P_{v_{2}}(k)(x)-k(x)\right) d \mu=-0.0268 & \text { Error }=5.5 \%, \\
\int_{[1,2]}\left(P_{v_{1}}(k)(x)-k(x)\right) d \mu=-0.0029 & \text { Error }=0.4 \%, \\
\int_{[1,2]}\left(P_{v_{2}}(k)(x)-k(x)\right) d \mu=-0.0327 & \text { Error }=4.3 \% .
\end{array}
$$

The conclusion of Example 5.3 is that, we must use the measure obtained with our procedure (the vector $v_{1}$ ) if we have a signal as $g$ that is affected by a perturbation as $r(x)$ and we are interested in the control of the area in the subintervals $[i-1, i]$, $i=1, \ldots, 6$. The approximation $P_{v_{1}}(k)$ is not better than $P_{v_{2}}(k)$ if we look at the global behavior of the function, but $P_{v_{1}}(k)$ is a better approximation to the function $g$ than $P_{v_{2}}(k)$ from an integral distance point of view.

Moreover, the main result of this paper, Theorem 4.2, states that we can use the same measure - the one defined by the vector $v_{1}$-if we want to study any other function as $h(x)=k(x)+g_{1}(x)$, where $g_{1}(x) \in S=\operatorname{span}\left\{f_{1}, \ldots, f_{6}\right\}+\operatorname{span}\left\{I, f_{1}, \ldots, f_{6}\right\}^{\lambda}$, and we get the same value for both $\epsilon_{o}$ and $\epsilon_{s}$ for $k(x)$. This means that we can use the same structure for the related Hilbert space defined by the vector $v_{1}$ for the approximation of functions as $h(x)$. We get good results (as good as in the case of $k(x))$ from the integral distance point of view.

\section{Conclusions}

We have developed a procedure to obtain different approximations to a function $g$ in a basis. This basis belongs to a structure that we have defined and we denote by $\lambda$ approximation structure. We have shown how we can use the properties of the family of norms that are defined in a $\lambda$-approximation structure in order to get a particular property of the approximation. The approximation we get in this way preserves the area in certain subsets $A_{i}$ of the support of the function, that is, the integral distance between the approximation and the original function in the sets $A_{i}$ is small.

Of course, it would be possible to use a procedure based on the minimization of a function of several variables (the error that we get in Section 3) to solve the stated problem. However, the results of Section 4 states that we can use the same vector that minimizes the symmetry error for the function $g$ in the case of a function $g+g_{1}$, where $g_{1} \in S$. This means that we can fix a particular metric for a Hilbert space and use it to find good approximations (with small integral distance) for each perturbation of $g$ as $g+g_{1}$. If we study our problem as a several variables problem we cannot get this general result.

We also would get a good approximation from the integral distance point of view by enlarging the finite set of orthonormal functions. In particular, if we define a $\lambda$ approximation structure with $N$ functions $\left\{f_{1}, \ldots, f_{n}\right\}$ and $N$ disjoint measurable sets 
$\left\{A_{i}: i=1, \ldots, n\right\}$, we would define another basis with functions of the set $\left\{f_{i} \chi_{A_{j}}\right.$ : $i, j=1, \ldots, n\}$. However, in this case we would find two problems.

(1) We cannot control the behavior of the approximated functions in the cohesion of the disjoint sets $A_{i}$. For instance, we cannot impose continuity to the approximation. Our method works with functions defined in the whole set $\Omega$.

(2) Our procedure needs $N$ functions. The use of the above mentioned set would give basis of $N^{2}$ functions.

Our technique may be used in several problems of function approximations and curve fitting. For example, it can be useful when we get a fit of a signal and we need to preserve the area of the original function in the channels defined for the signal. These kinds of problems are common in several experimental disciplines, as physical-chemistry, spectroscopy and nuclear physics. Further developments extending this theory to the discrete case can be used to fit histograms of experimental data to probability density functions. In this case,"preserving area" properties of the approximation is of clear interest.

\section{References}

[1] B. Beauzamy, Introduction to Banach Spaces and Their Geometry, 2nd ed., vol. 68, NorthHolland Mathematics Studies, no. 86, North-Holland Publishing Co., Amsterdam, 1985, Mathematical Notes. MR 88f:46021. Zbl 585.46009.

[2] G. P. Curbera, When $L^{1}$ of a vector measure is an AL-space, Pacific J. Math. 162 (1994), no. 2, 287-303. MR 94k:46070. Zbl 791.46021.

[3] B Banach space properties of $L^{1}$ of a vector measure, Proc. Amer. Math. Soc. 123 (1995), no. 12, 3797-3806. MR 96b:46060. Zbl 848.46015.

[4] J. Diestel and J. J. Uhl Jr., Vector Measures, no. 15, American Mathematical Society, Rhode Island, 1977. MR 56\#12216. Zbl 369.46039.

[5] R. B. Holmes, Geometric Functional Analysis and its Applications, no. 24, Springer-Verlag, New York, 1975, graduate texts in Mathematics. MR 53\#14085. Zbl 336.46001.

[6] H. E. Lacey, The Isometric Theory of Classical Banach Spaces, vol. 208, Springer-Verlag, New York, 1974, Die Grundlehren der mathematischen Wissenschaften. MR 58\#12308. Zbl 285.46024.

[7] D. R. Lewis, Integration with respect to vector measures, Pacific J. Math. 33 (1970), 157-165. MR 41\#3706. Zbl 195.14303.

[8] J. Lindenstrauss and L. Tzafriri, Classical Banach Spaces. I and II, Springer-Verlag, Berlin, 1996.

[9] S. Okada and W. J. Ricker, The range of the integration map of a vector measure, Arch. Math. (Basel) 64 (1995), no. 6, 512-522. MR 96e:46057. Zbl 832.28014.

[10] W. Rudin, Functional Analysis, McGraw-Hill Book Co., New York, 1973, McGraw-Hill Series in Higher Mathematics. MR 51\#1315. Zbl 253.46001.

L. M. García-Raffi: Departamento de Matemática Aplicada, Universidad Politécnica de Valencia, Camino de Vera, 14 46022, Valencia, Spain

D. Ginestar: Departamento de Matemática Aplicada, Universidad Politécnica de Valencia, Camino de Vera, 14 46022, Valencia, Spain

E. A. Sánchez-Pérez: Departamento de Matemática Aplicada, Universidad Politécnica de Valencia, Camino de Vera, 14 46022, Valencia, Spain 


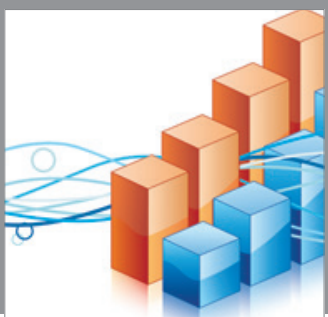

Advances in

Operations Research

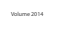

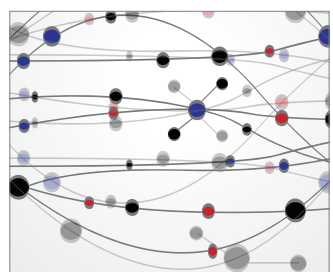

\section{The Scientific} World Journal
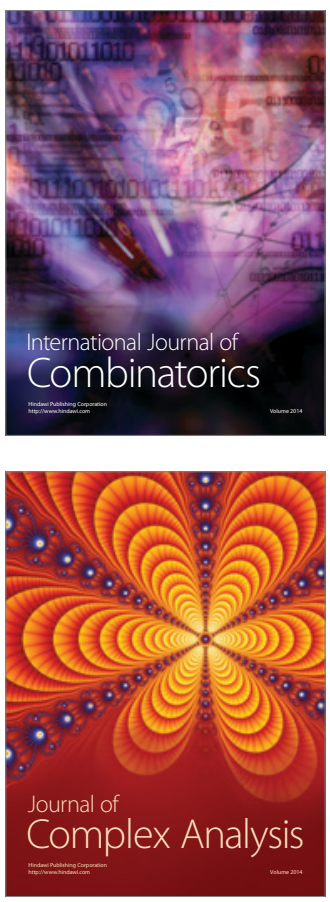

International Journal of

Mathematics and

Mathematical

Sciences
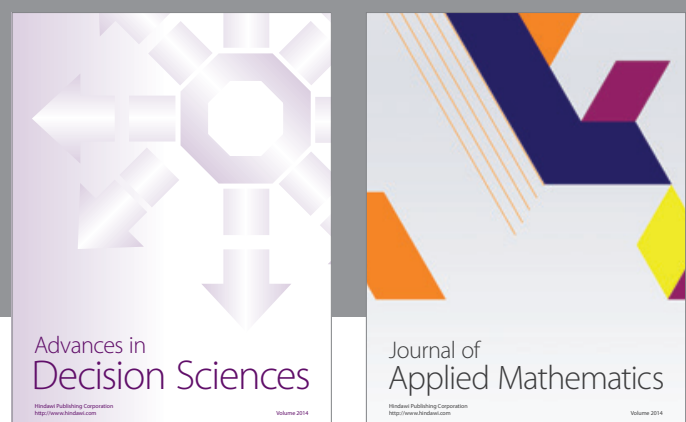

Journal of

Applied Mathematics
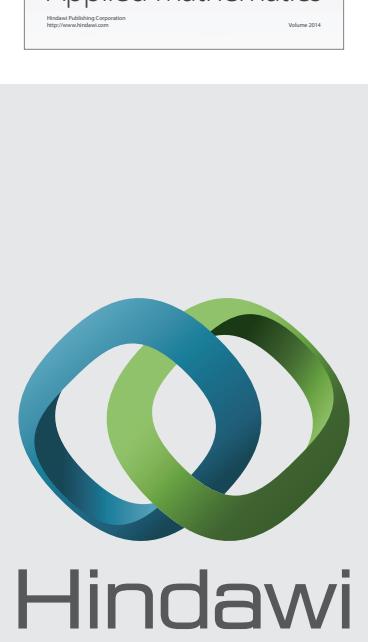

Submit your manuscripts at http://www.hindawi.com
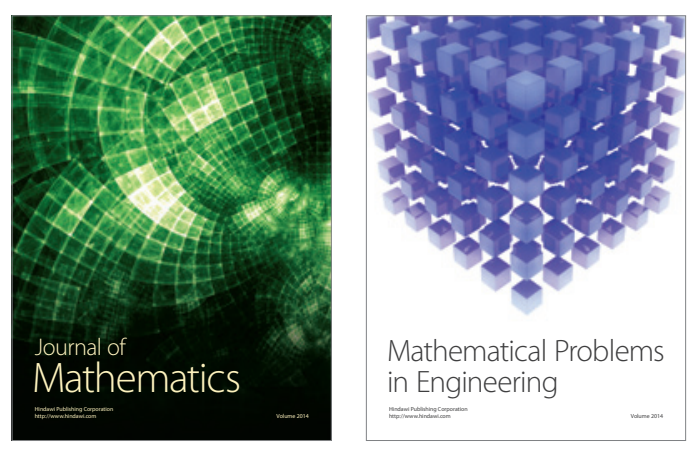

Mathematical Problems in Engineering
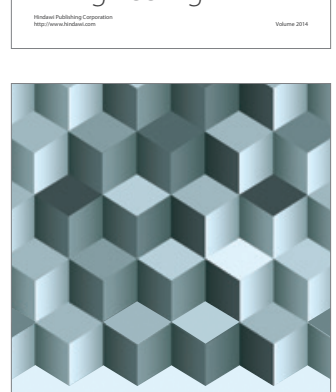

Journal of

Function Spaces
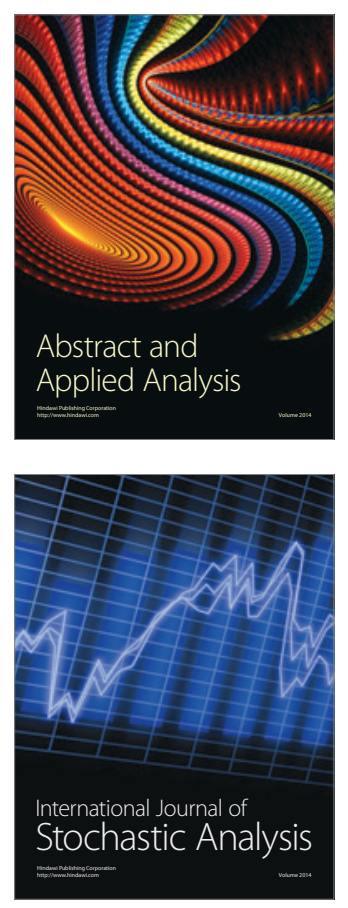

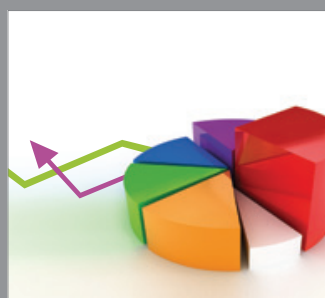

ournal of

Probability and Statistics

Promensencen
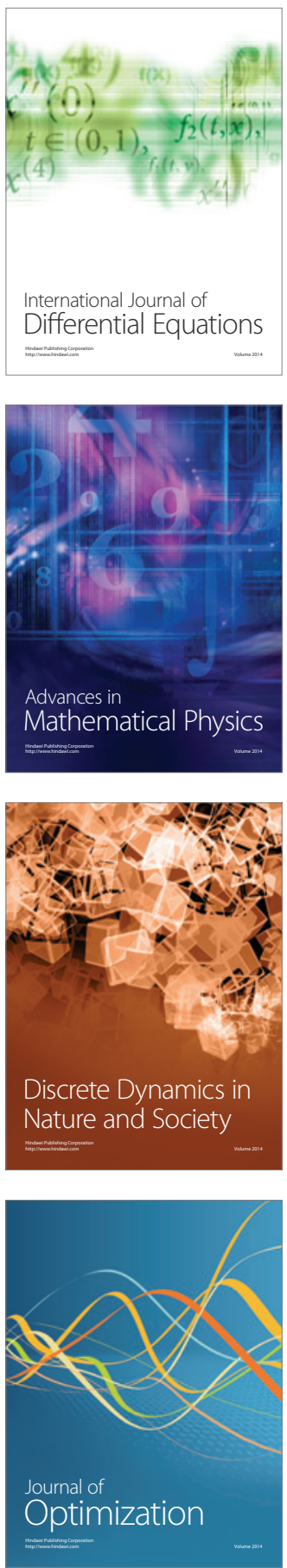